\title{
Análisis del fomento al Espíritu Emprendedor. Base de una Cultura Empresarial. Caso de estudio: Universidad de Pamplona.
}

ÁLVARO PARADA CARVAJAL 1 MARY LUZ ORDOÑEZ SANTOS²

\section{RESUMEN}

Dialogar de espíritu empresarial es hablar del corazón del desarrollo socioeconómico de las sociedades. Y es en ellas donde al promover una atmósfera de compromiso con el emprendimiento, se desarrolla un ambiente que estimula el surgimiento de nuevas empresas.

Este trabajo presenta el modelo que se ha ido conformando, en los últimos tres años en la Universidad de Pamplona, sin que se busque presentar un modelo educativo generalizado, éste sí es, el resultado del avance en el fomento de una cultura empresarial con la comunidad académica, el cual intenta hacernos capaces de identificar las soluciones a los problemas por los cuales atraviesa nuestra región, pues por no tener claridad sobre nuestro camino de desarrollo en ocasiones sólo zigzagueamos, en lugar de pensar seriamente sobre el papel protagónico que tenemos que desplegar dentro de nuestra responsabilidad social, para promover el desarrollo empresarial.

Inicialmente, se aborda el emprendimiento desde sus bases conceptuales, seguidamente, se caracterizan las fuerzas dinamizadoras de la propuesta de emprendimiento, a partir del momento que surgió la idea de crear un concurso interno de Planes de Negocio, cuyo objetivo fundamental es motivar, formar, asesorar y acompañar a los miembros de la comunidad académica de la Universidad de Pamplona, en la elaboración de planes que conduzcan a la creación de empresas. Finalmente, se explica el impacto que ha tenido en la academia, con la oportunidad que tiene la Universidad al formar profesionales integrales con una cultura empresarial, que comprendan las actitudes de perfeccionamiento individual, de creatividad, de innovación, de amor al trabajo, de aprender constantemente, de amor a la excelencia, de superación del temor al riesgo, etc. La universidad es un agente de cambio, principalmente a través de sus egresados.

\author{
Palabras Claves: \\ Emprendimiento. \\ Liderazgo. \\ Capital de Riesgo. \\ Emprendedor. \\ Desarrollo Empresarial.
}




\section{Analysis of the Foment free Entreprise Spirit. The Basis of the Business Culture. Case in Study: The University of Pamplona.}

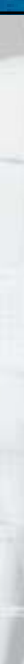

\begin{abstract}
To speak about the spirit of enterprise comes from the heart of the socio-economic development in society. It is here we find the promotion of an atmosphere of the promise to begin a business developing it within an ambience that stimulates the generation of new enterprises.

This work expresses the model constructed within the last three years at the University of Pamplona without wanting to present an educative design in general; this really is the result of the advancement together with the cultural fomenting of enterprise stemming from the academic community; this ambience intends to capacitate people to identify solutions to problems connected to our region. Since there is no real clarity for our developmental path, that zig-zag appears on our road instead of serious thought about the protagonistic role that should unfold in our social responsibility to promote the spirit of enterprise.

Initially, enterprise was touched upon from conceptual bases; then, it was characterized by dynamic forces in business proposals. From that moment the idea arose to create an internal contest called 'Plans for Business' whose fundamental objective was to motivate, form and assess as well as to accompany those members of the academic community here at the University of Pamplona in the elaboration of plans leading to the creation of new businesses. Finally, the impact of the academic environment with the opportunity the University offers to form integral professionals is explained here along with business culture including the attitudes of individual perfection, creativity, innovation, love for the work one does shown in constant learning and overcoming obstacles without fear of risking excellence, etc. The University is an agent of change, principally through its graduates.
\end{abstract}

${ }^{1}$ Dean of the Faculty of Economic and Enterprise Sciences University of Pamplona.

${ }^{2}$ Assistant Professor, Faculty of Economic and Enterprise Sciences, University of Pamplona.
Keys Words:

Entreprise.

Leadership.

Capital risk .

Businessman .

Enterprise development . 


\section{Bases conceptuales de la Cultura Empresarial.}
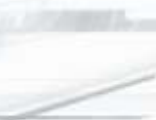

Desde el punto de vista conceptual, los investigadores explican el origen del término Espíritu Emprendedor en la palabra en francés entrepeneur. (Toro, et al 2003). A finales del siglo XVII y comienzos del XVIII, esta se refería a la persona que asumía una construcción civil, dirigía expediciones militares, a un guerrero que emprende una conquista 0 a otro tipo de aventureros. Posteriormente, el concepto fue enriqueciéndose con los aportes de economistas importantes Say (1852), Knight (1921), Baumol (1968 y 1993), Braudel (1985), Casson (1982) quienes diferenciaron al emprendedor del inversionista, al reconocer la diferencia en el rendimiento que cada uno espera de sus acciones $^{3}$.

En el contexto empresarial, muchos investigadores identifican al emprendedor como el individuo que ve y aprovecha la oportunidad, no teme arriesgar, tiene confianza y desarrolla una idea. De acuerdo con Shapero, lo que hay que encontrar es que las circunstancias internas y externas que rodean al individuo que va a ser empresario, produzcan en la persona un "desplazamiento" que le haga tomar la decisión de cambio en el estilo de vida, en la forma de ser, en la forma de pensar y de actuar.

Por otro lado McClelland, en su formulación teórica indica que existen tres tipos básicos de motivación: la motivación al logro, la motivación a la afiliación y la motivación al poder. En sus estudios encontró que hay una muy alta relación entre la gente que manifiesta alto nivel de logro y la gente que desempeña acciones empresariales.

Mientras los planteamientos de Ronstadt, respecto de la perspectiva empresarial, orientan hacia la necesidad de una educación que avive un pensamiento y una forma de proceder flexible y creativa en los discípulos y la comunidad en general, al evaluar con seriedad el efecto que el proyecto podría tener en el futuro sobre la vida.

Otro punto de partida, lo expone Allan Gibb quien manifiesta que la gente puede ser emprendedora en todas las situaciones de la vida siempre y cuando se presenten ciertas condiciones que el autor llama "las esencias de la Cultura Empresarial". Ellas son: independencia, libertad, capacidad de tener control sobre las decisiones que se toman, el sentimiento de compromiso y de responsabilidad.

Cabe destacar, que en el desarrollo de los Congresos Latinoamericanos sobre Espíritu Empresarial, se han mostrado experiencias de fomento de una cultura de emprendimientos, explicadas a partir de la nueva disciplina del Management y la puesta en práctica la mentalidad empresarial 4 .

${ }^{3}$ PEREIRA, L. F. (2003,10). Citado en Reflexión sobre algunas características del Espíritu Emprendedor.

${ }^{4}$ ANDREA, C. et. al. $(2002,22)$ Ponencia: Educación Empresarial: Visión de los estudiantes. XIII Congreso Latinoamericano sobre Espíritu Empresarial. 
Por otro lado, en nuestro país existe un incremento de esfuerzos para promover programas en Desarrollo de la Personalidad Emprendedora (ICESI, EAN, UPB, SENA) permitiendo caracterizar un fenómeno respecto a los atributos individuales y la futura inserción exitosa del profesional, en el mercado laboral.

Para entender algunas características del espíritu emprendedor colombiano, Pereira (2003) plantea una reflexión que permite identificar ciertos factores individuales dominantes, entre otros, la creatividad, la disposición a asumir riesgos moderados y las influencias culturales en la acción del emprendedor.

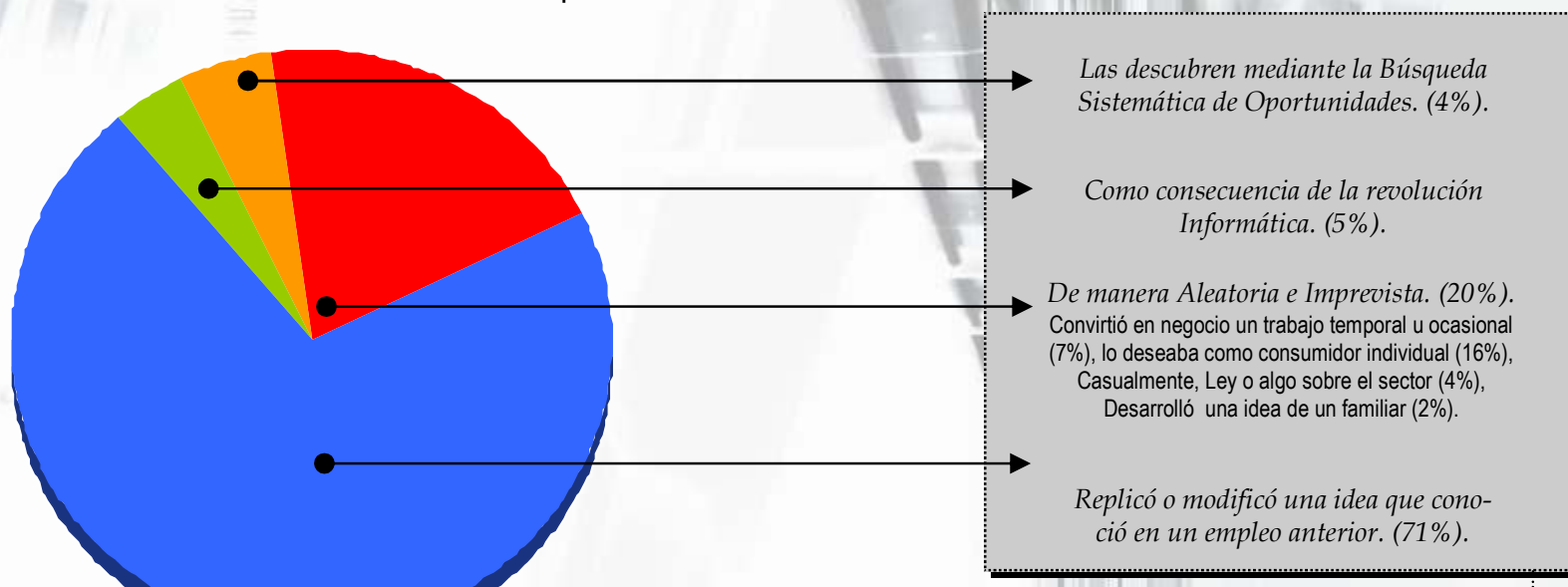

Fuente: 5 , Grafico $N^{\circ} 1$.

Entonces, la aplicación de una propuesta de educación en espíritu emprendedor, que permita a la Universidad de Pamplona, identificar factores propulsores claves, capaces de poner en marcha la construcción de una Cultura Empresarial, permitirá minimizar la brecha entre educación, productividad y desarrollo regional, como un reto al ofrecer al estudiante la oportunidad de obtener una experiencia como emprendedor y a la Universidad la oportunidad de integrarse a la comunidad acabando con el divorcio que tradicionalmente se da entre academia y sector productivo.

Ya hemos visto, que la educación empresarial es un problema de desarrollo de actitudes, motivaciones y orientaciones más que un problema específico de transmisión de información, sin que esto quiera decir que no exista contenido técnico. Es vital que se haga bajo una concepción de apertura mental, de liberalismo ideológico, de independencia de decisión. Por eso se necesita recurrir a metodologías de clase múltiples, a actividades dentro y fuera del aula, a experiencias prácticas, a talleres, a lecturas, a casos, a discusiones, a presentaciones, etc. En este contexto se encuentra que visión sin acción es sólo un sueño, y que acción sin visión carece de sentido. 


\section{Pilares básicos para el fortalecimiento del Emprendimiento en la Universidad de Pamplona.}

Nuestro marco general de análisis, para el fortalecimiento de competencias emprendedoras y de mentalidad empresarial en la Universidad de Pamplona, se basa en la premisa que en esta etapa se manifiesta la interacción del proceso de asesoría, el concurso de Planes de negocios Comprender y la Creación de un Fondo de capital semilla.

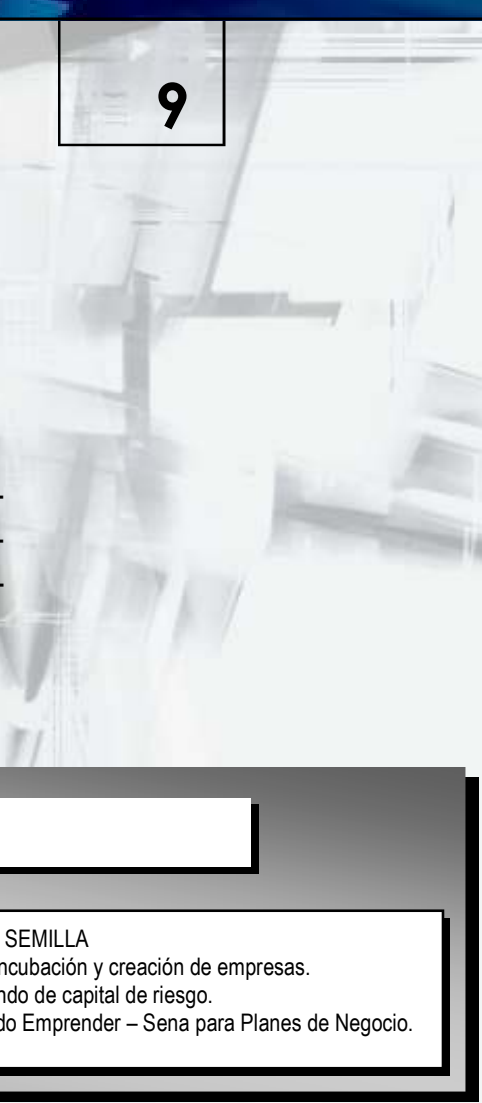

\section{Cuadro $N^{\circ} 1$.}

\section{Cátedra: Desarrollo del Espíritu Emprendedor}

A inicios de la primera década del siglo XXI, la Facultad de Ciencias Económicas, en respuesta al déficit empresarial que impedía ofertarle posibilidades de ocupación a sus egresados, empezó ha considerar el fomento del Espíritu Emprendedor como factor clave para la creación de empleo y mejora en la competitividad de los programas.

Este nuevo concepto entendido como el proceso mediante el cual las personas buscan oportunidades, satisfaciendo necesidades y deseos mediante la innovación, sin tomar en cuenta los recursos de los que disponen en el momento (Robbins, Coulter). Inculcó en el cuerpo profesoral el objetivo de crear una cultura que apoye y aliente la formación de un empresario innovador, que busca el cambio activamente al explotar oportunidades, motivado por la independencia al obtener una ganancia financiera.

En el camino por generar un ambiente empresarial, el pensum de 2000 hizo la inclusión de la asignatura "Desarrollo de la Personalidad Emprendedora", la cual tenía como propósito conseguir en el salón de clases un espacio de reflexión y participación que propiciará el fomento de nuevas ideas de negocio, mediante el reconocimiento del papel determinante de los individuos en la tarea de creación de empresas. Sin embargo, la transición de una mentalidad de empleados a la posibilidad de pertenecer a una sociedad de empresarios, era una idea que requería inicialmente apreciar los estilos individuales y sus más importantes fortalezas, buscando cualidades innatas que resultarán efectivas en futuras asociaciones de trabajo en equipo. 
El enfoque estimulaba a estudiar el entorno, a estar alerta a factores que pudieran ser el principio de un nuevo negocio; en general, los estudiantes reflejaban entrega y entusiasmo en el proceso de enseñanza, transmitiendo participación y compromiso. Mientras el desarrollo del nuevo curso daba lugar, nadie esperaba resultados rápidos, pues ello era un desafío más complejo.

Sin entrar en mayores consideraciones, la energía de un estudiante le impulsó a participar en el concurso nacional de Ventures 2002, no obstante la transición de un nivel local a otro nacional tuvo un impacto importante en el cuerpo profesoral al crear un estado de ánimo favorable hacia el proceso e incluso propició un momento crítico, al quedar de semifinalistas durante la selección.

Los diferentes puntos de vista generaron una crisis, que se convirtió en una oportunidad, al proponer adaptar la metodología de Ventures, al contexto local mediante el adelanto de un concurso de planes de negocios interno que permitiera asumir una relación activa y creativa en el desarrollo de habilidades emprendedoras, donde los estudiantes pudieran presentar proyectos específicos transmitiendo lo que querían desarrollar en su vida profesional.

En cierto sentido, este patrón de aprendizaje resaltaba la necesidad del trabajo interdisciplinario al permitirles a los estudiantes familiarizarse con la construcción de su propio papel de empresario.

\section{Proceso de asesoría y consultoría empresarial}

Este esquema empieza, considerando la consultoría y asesoría empresarial como el soporte que da acceso a la frontera del conocimiento, al recoger las apreciaciones de Christoper Curtis $^{6}$, que existen tres elementos fundamentales en la formación del Espíritu Empresarial, considerados cruciales, para implementar la propuesta: compromiso por parte del profesor, compromiso por parte del estudiante y trabajo conjunto de las universidades con los demás agentes económicos vinculados al desarrollo empresarial.

La gráfica No. 01 expresa de manera simplificada las áreas donde se requiere fortalecer el talento humano de las PyMes en Colombia, por ello la asesoría y consultoría dan aportes claves a los empresarios interesados en generar crecimiento y continuidad en sus empresas, al favorecer una actitud hacía los procesos de planeación, comunicación, innovación y competitividad. Si bien, en el país existe una oferta de opciones - muchas veces sin costo- en entidades especializadas en el fomento empresarial y la creación de empresas, desde Fundaciones, cooperativas, Universidades y Cámaras de Comercio, en nuestra región no se ha consolidado un programa de capacitación que ofrezca caminos de desarrollo. 


\section{Áreas donde se requiere fortalecer el Recurso Humano, gráfico №2.}

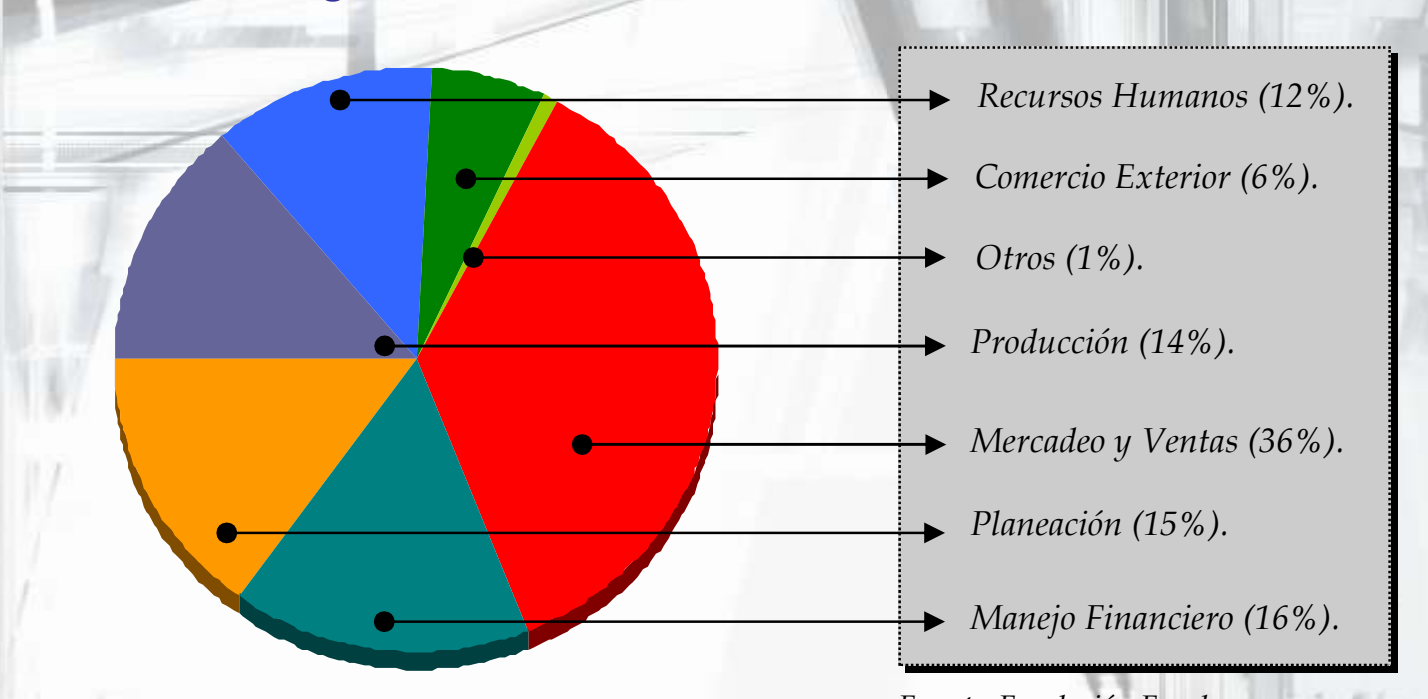

Fuente: Fundación Fundes.

Estudios realizados por la Fundación Corona, en Bogotá, demuestran que quienes reciben asesoría son aquellas empresas que generalmente les va mejor, tienen menos riesgos de cerrar y se les presentan mejores oportunidades de crédito y ventas.

Bajo esta perspectiva, conformar un centro de desarrollo empresarial en la Universidad ayuda a estimular la capacidad empresarial de los estudiantes y da una herramienta para registrar el desempeño de las empresas asesoradas. Cabe mencionar, que no obstante que FUNDES considera que los Servicios de Desarrollo Empresarial son la vía más adecuada para los evidentes problemas de gestión de las PyMes, un balance muestra bajos porcentaje de utilización de la pequeña y mediana empresa de la asistencia técnica, la capacitación y la consultoría.

Una herramienta de formación que utiliza el Sena en la actualidad para la capacitación y transferencia de conocimiento, es el trabajo en ambientes virtuales de aprendizaje AVA, denominado Pensamiento Empresarial el cual hace énfasis en la formación de mentalidad empresarial, planeación de empresa y Gestión.

La puesta en marcha de esta fase apunta al diseño de equipos de estudiantes de la Universidad como consultores, que establezcan sinergia con los empresarios en búsqueda del éxito del emprendimiento.

El cuadro No. 02 ilustra de la existencia de negocios formales en el Municipio de Pamplona, donde el desempeño de asesores-consultores puede reforzar comportamientos empresariales más productivos. 


\begin{tabular}{|c|c|}
\hline \multicolumn{2}{|c|}{$\begin{array}{c}\text { ACTIVIDAD ECONÓMICA } \\
\text { Negocios Formales } \\
\text { Municipio de Pamplona, cuadro } \mathrm{N}^{\circ} 2 .\end{array}$} \\
\hline Actividad económica / Negocios & $\%$ \\
\hline Cacharrería y Almacén en General ${ }^{7}$ & $22 \%$ \\
\hline Tienda & $16 \%$ \\
\hline Venta de Verduras y fruta & $12 \%$ \\
\hline Almacén de ropa & $11 \%$ \\
\hline Fabricación y venta de tejidos y Confecciones & $5 \%$ \\
\hline Salón de belleza & $5 \%$ \\
\hline Taller de mantenimiento en general ${ }^{8}$ & $4 \%$ \\
\hline Fama & $4 \%$ \\
\hline Cafetería y Restaurante & $4 \%$ \\
\hline Sala de internet - papelería y fotocopiadoras & $3 \%$ \\
\hline Otros $^{9}$ & $3 \%$ \\
\hline Panadería y bizcochería & $2 \%$ \\
\hline Droguería & $1 \%$ \\
\hline Almacén electrodomésticos & $1 \% \quad \mathrm{~V}$ \\
\hline Ferretería y venta de materiales de construcción & $1 \%$ \\
\hline Billares y juegos & $1 \%$ \\
\hline Consultorios y Laboratorios & $1 \%$ \\
\hline Licorería & $1 \%$ \\
\hline Floristería & $1 \%$ \\
\hline Fotografía & $0 \%$ \\
\hline Funeraria & $0,3 \%$ \\
\hline Ladrillera & $0,2 \%$ \\
\hline Total & $100 \%$ \\
\hline
\end{tabular}

Fuente: Primer Censo Socio-empresarial del Municipio de Pamplona 2004

Grupo de investigación UNIR- Universidad-Región y Cámara de Comercio Pamplona.

El programa denominado "Estudiantes como consultores" consiste en invitar a un empresario a participar en un programa de mejoramiento continuo, luego de un proceso de sensibilización y socialización se facilita el acercamiento empresario-estudiante. Éste va a la empresa a estudiar los resultados en áreas funcionales y califica cada una de las áreas administrativas, luego el empresario acude a la Universidad a conocer las áreas susceptibles de mejoramiento y construyen un plan de acción conjuntamente entre empresario, colaboradores, estudiante y docentes asesores.

${ }^{7}$ Cacharrería y almacén en general: incluyen establecimientos de comercio en venta de juguetes, adornos, accesorios, y víveres.

¿Taller de mantenimiento en general: incluye los negocios de reparaciones mecánicas, computadores, electrodomésticos y bicicletas.

${ }^{9}$ Otros negocios: incluye los negocios de marquetería, carpinterías, venta de chance y artículos religiosos. 


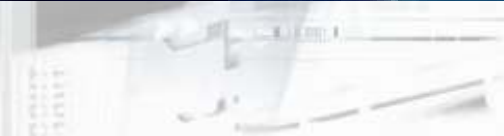

En una primera fase se han realizado contacto con 71 unidades económicas del sector productivo, espacio que ha constituido la mayor evidencia de proyección y pertinencia social de los programas de la Facultad de Ciencias Económicas y Empresariales.

Una segunda fase, ha permitido establecer un compromiso con el desarrollo del sector socioeconómico, en el marco del convenio con DANSOCIAL, acompañando la organización y funcionamiento de nuevas formas asociativas que contribuyan al mejoramiento de las condiciones de vida de la comunidad, atendiendo los sectores de: producción frutícola y hortícola; en convenio con la Agencia de Cooperación Alemana para el Desarrollo GTZ (manejo sostenible de bosques: cadena productiva del recurso forestal); red local de distribución de productos de consumo masivo, conformada por tenderos y pequeños comerciantes y puntos de atención de servicios básicos estudiantiles ${ }^{10}$.

Hoy en día, la imagen del emprendedor está asociada a generar ideas de negocio rentables para su propia empresa. En el mejor de los casos concebir ideas enfrenta a los emprendedores a abrir los ojos, a buscar experiencias diferentes que les permiten crecer. Con una maleta llena de sueños, se comienza a tratar de darle forma a un proyecto.

Al principio la labor es más difícil de lo que se piensa, pues pocos están realmente dispuestos a comprometerse con el proceso de investigación que hay detrás de cada idea. A través del análisis del comportamiento de los planes de negocio, en este trabajo vemos como el entusiasmo por crear valor a una idea reúne equipos multidisciplinarios intentando dominar un tema.

La rentabilidad engrana, en la segunda variable, de la cual quiere apropiarse el emprendedor; reportar beneficios puede motivarle a dedicarse con pasión a la idea. En la mayoría de "creadores de ideas de negocios" el fortalecimiento financiero se idealiza con rapidez.

En el interés de aportar una luz a los jóvenes universitarios con características emprendedoras e impulsar el crecimiento económico, se diseñó como pilar fundamental: el Concurso de Planes de Negocios COMPRENDER, herramienta que daría un marco de trabajo en la etapa inicial, como fuerza dinamizadora del contexto interno para obtener respuesta de los miembros de la Institución.

El 1 de marzo de 2004, comenzó la primera fase del Concurso COMPRENDER que tenía como propósito la construcción de una cultura empresarial que promoviera el espíritu y la actitud emprendedora como estrategia para el desarrollo socioeconómico de la región y el país.

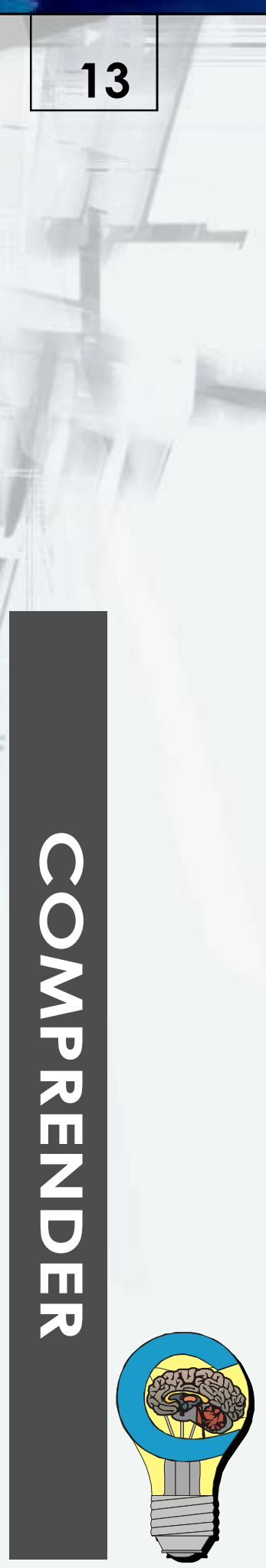


Reglamentado por el Centro de Prácticas de la Facultad de Ciencias Económicas, se lanzaba el concurso de Planes de Negocio cuya premisa básica sería la innovación y calidad que tuvieran los equipos al tener la capacidad de traducir las ideas y descubrimientos en actividad productiva - empresa.

La idea permitía participar profesores, estudiantes regulares de los programas vigentes y egresados de la Universidad de Pamplona, que de forma individual o por grupos presentaran ante la organización del Concurso COMPRENDER, resúmenes ejecutivos de proyectos que representarán un potencial impacto en el ámbito local, nacional e internacional.

Entre las fases del Concurso, se decidió ofertar a los participantes una serie de seminarios de capacitación con el objetivo de desarrollar habilidades y mejorar las competencias de los miembros en una atmósfera de responsabilidad y participación que permitiera crear vínculos estratégicos entre ideas de negocios análogas.

\section{Ideas de negocios inscritas en Comprender}

En un terreno inédito para la comunidad universitaria, el Concurso de Planes de Negocios 2004, se convirtió en una alternativa importante para 57 ideas.

El 70\% de los planes procedían de estudiantes de la Facultad de Ciencias Económicas quienes en el aula habían reconocido que hacer empresa era un buen comienzo para su carrera.

De otra parte, la facultad de Ingenierías y Arquitectura participaba con el $9 \%$ de ideas de negocio provenientes de equipos que investigan, diseñan y adaptan tecnologías con el ánimo de tener un producto final distribuible en el mercado.

Con un $7 \%$ de planes de negocios participaban egresados, quienes después de haber logrado un título universitario, querían incursionar en el camino de crear su propia empresa.

La Facultad de Artes y Humanidades y la Facultad de Educación participaron con un $5 \%$ de ideas a favor de comunidades, buscando garantizar la sostenibilidad de sus proyectos.

Docentes de la Facultad de Ciencias Básicas participan con un 4\% de ideas de negocio, al buscar demostrar que su experiencia es una fortaleza al asumir el reto de crear empresa. 


\section{FACE}

CONCURSO DE PLANES DE NEGOCIO COMPRENDER, cuadro N ${ }^{\circ} 3$.

\begin{tabular}{|c|c|c|c|c|c|c|c|c|c|}
\hline & CONC & JRSO DE PI & NES DE & EGOCIO & $\mathrm{CON}$ & PRENL & DER, cue & dro N 3 & \\
\hline & Procedencia & F. C. E. E. ${ }^{11}$ & F. A. H. ${ }^{12}$ & F. C. B. ${ }^{13}$ & F. I.14 & F. E. 15 & F. C. A. ${ }^{16}$ & Comunidad & Egresados \\
\hline \& & $\begin{array}{l}\text { Ideas de negocios } \\
\text { Inscritas }\end{array}$ & 40 & 3 & 2 & 5 & 3 & 0 & 0 & 4 \\
\hline & Participantes & 84 & 5 & 2 & 20 & 6 & 0 & 0 & 4 \\
\hline 농 & $\begin{array}{l}\text { Ideas de negocios } \\
\text { Inscritas }\end{array}$ & 24 & 1 & 1 & 9 & 0 & 1 & 5 & 2 \\
\hline & Participantes & 47 & 1 & 2 & 15 & 0 & 2 & 4 & 2 \\
\hline
\end{tabular}

Fuente: Centro de Prácticas de la Facultad de Ciencias Económicas de la Universidad de Pamplona.

\section{Naturaleza de los Proyectos}

Un pilar de la economía regional es la agricultura y precisamente en este renglón se concentran un 54\% de las ideas de negocio; básicamente su estrategia es conseguir la producción y comercializar los productos, no obstante algunos plantean procesar las frutas, verduras y derivados de la leche y así obtener otros beneficios.

Si bien la cultura del consumo orgánico todavía no existe en la región, unos emprendedores esbozan aprovechar la posición geográfica de sus lugares de origen, para sacar adelante proyectos con tomate, duraznos y cacao.

De otro lado, son menores las propuestas orientadas a trabajar con animales: ganado y cerdos, como negocios con potencial interés emprendedor. En una ciudad universitaria donde es latente la presencia de jóvenes, incubar ideas de negocios que ofrezcan servicios a los estudiantes es oportuno, por ello un $28 \%$ de planes presentados en COMPRENDER se concentran en ofertar servicios de alimentación, alojamiento, arreglo de ropa, asesorías académicas, diversión, organización de eventos, centrando su mayor fortaleza en el servicio al cliente.

Novedosas ideas de negocio soportadas en tecnología ocupan el $11 \%$, estos proyectos buscan incluir en sus productos finales transferencia tecnológica.

\section{Premios y reconocimientos del Concurso Comprender}

Establecer cuál es el plan de negocios con mayor nivel de calidad, en un momento en que la comunidad universitaria empezaba a interesarse por el tema de la cultura em-

${ }^{11}$ F. C. E. E: Facultad de Ciencias Económicas y Empresariales.

${ }^{12}$ F. A. H: Facultad de Artes y Humanidades.

${ }^{13}$ F. C. B: Facultad de Ciencias Básicas.

${ }^{14} \mathrm{~F}$. I: Facultad de Ingenierías.

${ }^{15}$ F. E: Facultad de Educación.

16F. C. A: Facultad de Ciencias Agrarias. 
prendedora, era un dilema. Una forma de tomar la decisión fue establecer tres categorías: 1) Premio al Entusiasmo Emprendedor 2) Premio al Compromiso Emprendedor 3) Reconocimiento al Emprendimiento

La participación durante los seminarios de capacitación realizados durante el Concurso fue el criterio más importante al reportar 7 ideas de negocio con el "Premio al Entusiasmo Emprendedor". Ante la revisión de los profesores-asesores, 7 proyectos con unidad en la estructura administrativa y estudio de mercado recibieron el "Premio al Compromiso Emprendedor"

Y el informe final, señaló 12 ideas de negocio meritorias del "Reconocimiento al Emprendimiento" con un premio de dos millones de pesos $(\$ 2.000 .000)$ que les permitiría demostrar que con trabajo los sueños son metas alcanzables.

\section{Ganadores en el Concurso Nacional Emprender: Nace una empresa en la Universidad de Pamplona.}

El Fondo Emprender-SENA avaló el Plan de Negocios "Distribuidora Campesina de Productos Agrícolas de la Región de Pamplona -DISTRICAMPAM, presentado por cinco estudiantes de la Facultad de Ciencias Económicas y Empresariales. El plan de negocios fue escogido junto con otros 349 proyectos de un total de 6.480 que se formalizaron a nivel nacional, ante el Fondo Emprender en la Segunda Convocatoria realizada en el 2005.

Para la ejecución y puesta en marcha del negocio el grupo de estudiantes podrá recibir ochenta millones de pesos $(\$ 80.000 .000)$ de los recursos que asigne el Fondo Emprender deberán ser utilizados para la financiación del capital de trabajo del plan de negocio, entendiéndose por capital de trabajo, los recursos necesarios para el funcionamiento y operación de la empresa consistente en dinero destinado al pago de salarios, insumos para el ciclo productivo que contribuyen directamente o que forman parte del proceso de producción; adquisición de maquinaria y equipo, costos que se generen por concepto de constitución legal y licencias requeridas por ley para el normal funcionamiento del proyecto.

Este plan de negocios inicialmente hizo su tránsito en el concurso interno de planes de negocios COMPRENDER 2004. En esa oportunidad fueron seleccionados y favorecidos con un premio de dos millones de pesos (\$2.000.000), lo que fijó un rumbo hacia delante, así que al llegar a este punto se decidió articularlo al concurso nacional del Fondo EMPRENDER-SENA.

La obtención de una fuente de financiamiento para gestionar el emprendimiento concebido en el plan de negocios, perfecciona en el grupo de profesores-asesores el enfoque de las iniciativas y las modalidades para la gestión, lo cual constituye una valiosa experiencia de aprendizaje que permitirá avanzar rápidamente y con determinación en futuros compromisos empresariales para impulsar el espíritu emprendedor en los miembros de la comunidad académica.
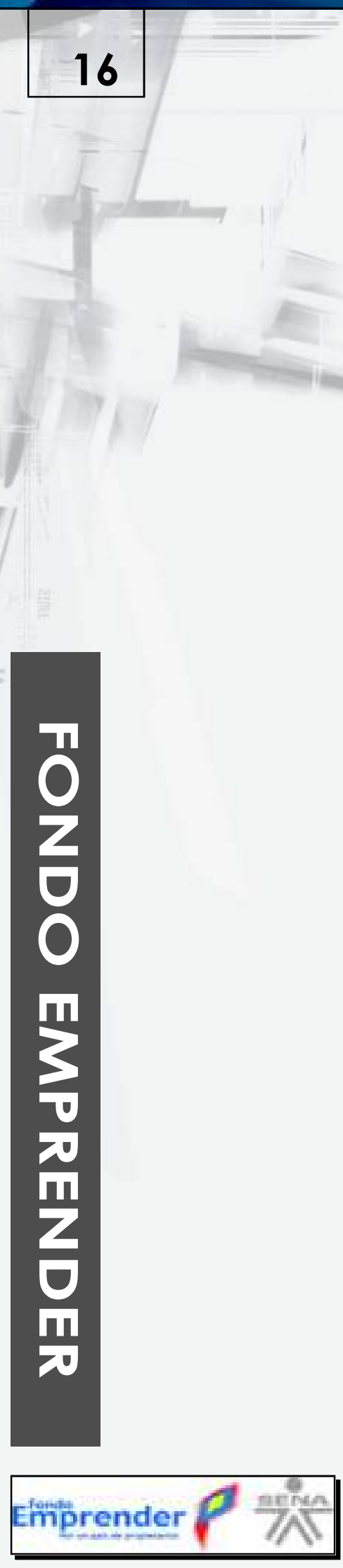


\section{Una Idea de Negocio que nuestros estudiantes volvieron realidad}

La Facultad de Ciencias Económicas y Empresariales de la Universidad de Pamplona promueve la cultura del emprendimiento en la región, mediante la constitución y desarrollo de nuevas empresas.

Los primeros beneficiaros directos, de la promoción de una mentalidad emprendedora en la Facultad de Ciencias Económicas y Empresariales son Sonia Yadid Hernández Hernández de Charta (Santander) y Franklin Geovanny Vargas Capacho de Cúcuta, estudiantes de Administración Comercial y de Sistemas de la Universidad de Pamplona.

Inicialmente, no pensaron que aquello que empezó, hace 3 años, como una idea de negocio, para cumplir con un trabajo académico, hoy, es un proyecto premiado a nivel nacional por el Fondo Emprender-Sena.

\section{EL SUEÑO}

"El amor por la tierra y los productos que ella nos da, comenzó desde que estudiaba como bachiller en la modalidad agropecuaria" recuerda Sonia. Mientras su socio Franklin confiesa que el amor al campo está ligado a sus orígenes familiares. La pasión por el agro regional, les hizo soñar con una empresa que contribuyera al desarrollo regional mediante la distribución de productos agrícolas de la provincia de Pamplona.

El esquema de trabajo de DISTRICAMPAM les permitirá a los productores vincularse, a cambio de la comercialización de un promedio de 50 productos entre hortalizas, tubérculos, verduras y frutas.

Fresas, mora, papa, brócoli, y otros productos de la región, serán entregados en tiendas, comedores estudiantiles y restaurantes, con un valor agregado en la presentación, limpieza y mejor calidad en la selección; esperan ser el boom en la ciudad, teniendo en cuenta los nuevos hábitos del consumidor hacia lo mejor y más natural. Esperan que los compradores empiecen a valorar más la relación costo/beneficio en los productos alimenticios que les den.

Arrancando les ha tocado de "toderos", han tenido que aprender de cultivos, cosechas, empaques, distribución, pero están jugándosela toda por el reto de conquistar el mercado. "A futuro quieren fortalecer su operación de distribución y la logística en el manejo de productos tan perecederos", asegura Franklin.

La experiencia de aproximarse a la realidad empresarial, les ha enseñado que se requiere de mucha disciplina, tiempo, asesoría y fuertes inversiones para conformar empresa. En nuestro caso, el efecto de aplicar un modelo de creación de empresas en el programa académico, ya comienza a notarse, afirman los emprendedores.

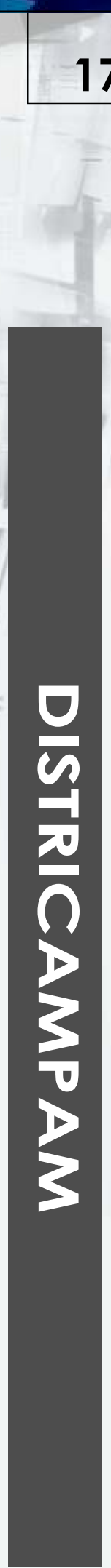




\section{CONCLUSIONES}

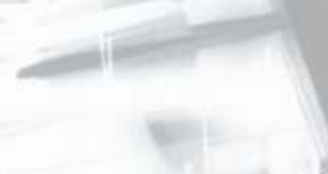

Un entorno económico globalizante y de alta competitividad, exige a las universidades proponer modelos creativos, que impulsen nuevos negocios que generen bienestar y riqueza, a partir del conocimiento, además demanda que sus egresados sean ciudadanos emprendedores. Si bien, alcanzar estos objetivos implica acciones en el sector educativo universitario, también se pueden desarrollar proyectos particulares en el nivel secundario y nivel primario y, además, en el núcleo familiar, en la pretensión de incidir notablemente en la formación de una cultura empresarial.

Con lo anterior, la exigencia no nos puede llevar al pecado imperdonable de copiar modelos, conceptos, e ideas que en otros ámbitos seguramente son buenos, pero en los propios podrían no serlo. Entonces, se debe trabajar activamente en modificar el ambiente que facilite el surgimiento del empresario, estableciendo organismos de apoyo, fuentes de financiamiento, cuerpos de asesores, identificación de oportunidades, entre otros.

Los concursos estimulan a los jóvenes a tener su propia idea de negocio, al percibir que al tener una actividad empresarial tendrá un gran beneficio no sólo personal sino también social, al convertirlos en modelos para los demás, originando en ellos sentimientos de mayor credibilidad. La experiencia ha permitido comprobar que los jóvenes encuentran en el fomento del Espíritu empresarial un complemento importante de sus otras actividades académicas, además encuentran la diferencia entre educación empresarial y educación gerencial, la primera da prioridad a la persona y la complementa con el quehacer, mientras que la segunda se queda en el quehacer.

Realizado el análisis al fomento del espíritu emprendedor, queda la convicción de difundir las ideas fundamentales del concepto de desarrollo socioeconómico basado en el espíritu empresarial, motivando a los miembros de la comunidad universitaria e integrando este concepto a los procesos académicos para crear una cultura que aprecie y apoye el espíritu empresarial.

\section{BIBLIOGRAFÍA}

Corporación Innovar. (1999). Seminario Empretec: Programa para el desarrollo de habilidades emprendedoras. Bogotá: Organización de Naciones Unidas.

De Bono, E. (1996). Ideas para profesionales que piensan. Bogotá: Paidós.

HARVARD BUSINESS REVIEW. (1999). Iniciativa Emprendedora. Ediciones Deusto.

GIBBS A. (1987). Entreprise culture: Its meaning and implications for education and training. Journal of European Training. M.C.B. Publication Spring.

RONSTADT, R.C. (1984). Entrepreneurship: Text, case and notes. Lord Publishing. Dover, Mass.

SHAPERO, A. (1982). Some Social Dimension of Entrepreneurship. Encyclopedia of entrepreneurship. Prentice Hall, Englewood Cliffs.

VARELA V. R. (1990). Las características empresariales y su desarrollo educacional. Memorias IV Congreso Latinoamericano sobre Espíritu Empresarial. Publicaciones ICESI, No. 37. Octubre-diciembre. 